ORIGINAL RESEARCH

\title{
Patient Outcomes Cost, and Length of Stay Associated with Biventricular Assist Devices in the United States (2009- 2015)
}

Hyeon-Ju Ryoo Ali ${ }^{1}$, Afshin Ehsan ${ }^{2}$, Kevin Kennedy ${ }^{3}$, Peter Riley ${ }^{4}$, Angie Seo ${ }^{5}$, Navkaranbir S Bajaj $^{5}$, Amitoj Singh ${ }^{6}$, Frank Sellke ${ }^{2}$, Daniel Levine ${ }^{7}$, Michael Kiernan ${ }^{8}$ and Rayan Yousefzai ${ }^{1^{*}}$

${ }^{1}$ Department of Cardiology, Houston Methodist Hospital, Houston, USA

${ }^{2}$ Division of Cardiothoracic Surgery, Lifespan Cardiovascular Institute and The Warren Alpert Medical School of Brown University, Providence, RI, USA

${ }^{3}$ Mid-America Heart Institute, St. Luke's Health System, Kansas City, MO, USA

${ }^{4}$ Division of Cardiology, University of Maryland, Baltimore, MD, USA

${ }^{5}$ Division of Cardiology, Mount Sinai Hospital, New York City, USA

${ }^{6}$ Division of Cardiovascular Disease, University of Alabama, Birmingham, AL, USA

${ }^{7}$ Division of Cardiology, University Medical Center Tucson, Tucson, AZ, USA

${ }^{8}$ Division of Cardiology, Lifespan Cardiovascular Institute and The Warren Alpert Medical School of Brown University, Providence, RI, USA

${ }^{9}$ Division of Cardiology, Tufts Medical Center, Boston, MA, USA

*Corresponding author: Rayan Yousefzai, MD, Department of Cardiology, Houston Methodist Hospital, 6550 Fannin St, Suite 190, Houston, TX 77030, USA, Tel: 713-441-1100
Abstract
Objectives: With recent advances in left ventricular assist device (LVAD) technology, the utilization of LVADs has increased while mortality, cost, and length of stay (LOS) have significantly decreased. This study sought to determine the national trends of surgically implanted biventricular assist devices (BiVADs).
Methods: This is a retrospective analysis of the National Inpatient Sample (NIS) database for internal BiVADs ( $\mathrm{n}=$ 538) and external BiVADs ( $n=727$ ) from 2009 to 2015.
Results: Utilization of all surgically implanted BiVADs decreased $(4.8 \%$ per year, $p<0.001)$, and was driven by a decline in utilization of external BiVADs (9.4\% per year, $p<0.001)$. There was no significant change in BiVAD- associated mortality. However, cost and LOS increased significantly, specifically among patients who died during their hospitalization. Notably, the burden of comorbidities among BiVAD recipients increased during the study period. Compared to external BiVAD patients, those with internal BiVADs incurred higher costs, had longer LOS, but were more likely to survive.

Discussion: The surgically implanted BiVAD outcome and utilization trends are less favorable compared to surgically implanted LVAD. Future studies and registries are needed to optimize BiVAD management and patient selection to improve the patient outcome and cost-efficiency.

\section{Keywords}

Biventricular assist device, Outcomes, Cost, Length of stay
Abbreviations
BiVADs: Biventricular Assist Devices; CAD: Coronary Artery Disease; CF: Continuous-Flow
GI: Gastrointestinal; NIS: National Inpatient Sample; LOS: Length of Stay; LV: Left Ventricle; LVAD: Left Ventricular Assist Device; MCS: Mechanical Circulatory Support; MI: Myocardial Infarction; PF: Pulsatile-Flow; RV: Right Ventricle; TAH: Total Artificial Heart; TIA: Transient Ischemic Attack; VAD: Ventricular Assist Device of Stay Associated with Biventricular Assist Devices in the United States (2009-2015). Int J Clin Cardiol 8:249. doi.org/10.23937/2378-2951/1410249

Accepted: February 17, 2022: Published: February 19, 2022

Copyright: (C) 2022 Ali HJR, et al. This is an open-access article distributed under the terms of the Creative Commons Attribution License, which permits unrestricted use, distribution, and reproduction in any medium, provided the original author and source are credited. 


\section{Introduction}

From 2009 to 2015, in-hospital mortality and complication rates associated with left ventricular assist device (LVAD) implantation significantly declined [1,2]. Much of the favorable outcomes have been attributed to the evolution of mechanical circulatory support from pneumatic mechanical-bearing pulsatile-flow (PF) pumps to magnetically-levitated continuous-flow (CF) devices. According to the latest results from the MOMENTUM 3 trials, 77\% of patients receiving the Heart Mate III LVAD had a stroke-free 2-year survival [3]. Despite these encouraging outcomes, right ventricular failure (RVF) post-LVAD remains a significant cause of adverse outcomes - in some instances requiring biventricular assist devices (BiVADs) [4].

Despite the advances in LVAD technology, most BiVADs - including total artificial hearts (TAHs) and external BiVADs - are still pneumatic PF devices. BiVAD configurations are diverse and include completely internalized devices - such as the TAH or two separate internal ventricular assist devices (VADs) - and external support, such as paracorporeal PF BiVADs, and CF centrifugal pumps (e.g.,CentriMag) [5]. Given these fundamental differences, it is unknown whether the promising national trends in the LVAD population -improvement in survival, decrease in length of stay (LOS), and reduction in complication rates - also hold true for patients receiving surgically implanted BiVADs. This study is the first to describe the national trends in utilization, mortality, complications, cost, and LOS for patients receiving BiVADs.

\section{Methods}

\section{Study design}

This is a retrospective analysis of the National Inpatient Sample (NIS) database. The NIS is reflective of all hospital admissions in the United States. It serves as an essential tool for analyzing the trends in outcomes and costs of healthcare procedures. More details regarding the database are available online [5]. Given the data from NIS are de-identified, this study was exempt from review by our Institutional Review Board.

\section{Study population and outcomes}

The NIS database was queried to identify the hospitalizations of the following population: 1) Adult patients age $\geq 18$ years old and 2 ) Those with implantation of surgically implanted internal BiVADs (International Classification of Disease $9^{\text {th }}$ revision (ICD-9) code 37.52, which includes TAH) orsurgically implanted external BiVADs (ICD-9 code 37.60). The patients with orthotropic heart transplantations (ICD-9 code 37.5, 37.51, and 33.6), temporary non-implantable external systems (ICD-9 code 37.62), single ventricular devices (ICD-9 code 37.65 and 37.66 ), as well as percutaneous external devices (ICD-9 code 37.68) were excluded. Examples of external BiVADs include CentroMags implanted in LV and RV, as well as Thoratec VAD in LV and RV. Examples of internal BiVADs include Syncardia and Jarvik 2000. Of note, the use of two separate surgically implanted VADs used for biventricular support fell under ICD coding for "single ventricular devices" and thus could not be captured in our analysis. We also excluded the patients receiving intra-aortic balloonpumps (IABPs, ICD-9 codes 37.61 and 37.68 ) as well as implantable heart systems (ICD-9 code 37.66). In total, 1,264 patients met these criteria, representing 21,204 patients nationwide.

Primary outcomes of interest were utilization and in-hospital mortality. Secondary outcomes included LOS, cost of hospitalization, and complications: Major bleeding requiring transfusion, gastrointestinal (GI) bleed, hemoperitoneum, intracranial bleed, hemolytic anemia, vascular complications, acute renal failure, mechanical complications, systemic embolism, stroke, transient ischemic attack (TIA), and infections. In addition to demographic information, other relevant comorbidities (e.g., coronary artery disease (CAD), myocardial infarction (MI), chronic pulmonary disease, anemia, coagulopathy, diabetes, hypertension, renal failure, and peripheral vascular disease) were queried. Information regarding the hospitalization -the region of hospital, location and teaching status, volume, elective vs. non-elective implantation, and insurance statuswere included in the analyses.

\section{Statistical analysis}

The baseline characteristics of patients with internal and external BiVAD were compared using the $\chi^{2}$ test for categorical variables and analysis of variance for continuous variables. The Poisson regression model was used to assess the change in estimated quarterly volumes of internal, external, and all BiVADs. Finally, we sought to explain the national trends using exploratory subgroup analyses. The total population was divided into patients who survived the index hospitalization and those who died during the hospitalization. We performed regression analyses using comorbidities and complications as covariates. STATA was used to perform all analyses. Statistical significance was considered to be $\mathrm{p}<0.05$.

\section{Results}

A total of 21,204 patients received either surgically implanted internal or external BiVADs between 2009 and 2015. The mean age of the population was 51.2 years (standard deviation or SD $=14.4$ ) at the time of implantation (Table 1). Approximately one quarter (25.1\%) of all patients were female, and two-thirds (63.7\%) were Caucasians. Almost half of the patients (48.4\%) had private insurance, and $30.9 \%$ had Medicare coverage. Most implantations took place in large (86\%), urban teaching hospitals (90.3\%), and $18.5 \%$ of them occurred as elective admissions. 
Table 1: Demographics and hospitalization characteristics for all BiVADs.

\begin{tabular}{|c|c|}
\hline & All BiVADs $(n=1264)$ \\
\hline Age, years (standard deviation) & $51.2(14.4)$ \\
\hline Female, n (\%) & $317(25.1 \%)$ \\
\hline \multicolumn{2}{|l|}{ Race, n (\%) } \\
\hline White & 719 (63.7\%) \\
\hline Black & $190(16.8 \%)$ \\
\hline Hispanic & 95 (8.4\%) \\
\hline Asian or Pacific Islander & $40(3.5 \%)$ \\
\hline Native American & $10(0.8 \%)$ \\
\hline Other & 75 (6.7\%) \\
\hline Missing & $136(10.8 \%)$ \\
\hline \multicolumn{2}{|l|}{ Comorbidities, n (\%) } \\
\hline Coronary artery disease & $445(35.2 \%)$ \\
\hline Myocardial infarction & 393 (31.1\%) \\
\hline Chronic pulmonary disease & $112(8.9 \%)$ \\
\hline Iron deficiency anemia & $172(13.6 \%)$ \\
\hline Chronic blood loss anemia & $15(1.2 \%)$ \\
\hline Coagulopathy & $606(47.9 \%)$ \\
\hline Diabetes & $224(17.7 \%)$ \\
\hline Hypertension & 376 (29.7\%) \\
\hline Hypothyroidism & $54(4.3 \%)$ \\
\hline Liver disease & $50(3.9 \%)$ \\
\hline Lymphoma & $34(2.7 \%)$ \\
\hline Fluid and electrolyte disorders & $791(62.6 \%)$ \\
\hline Metastatic cancer & $5(0.4 \%)$ \\
\hline Obesity & $128(10.1 \%)$ \\
\hline Peripheral vascular disorders & $122(9.7 \%)$ \\
\hline Pulmonary circulatory disorders & $38(3.0 \%)$ \\
\hline Renal failure & $311(24.6 \%)$ \\
\hline Valvular disease & $52(4.1 \%)$ \\
\hline \multicolumn{2}{|l|}{ Insurance, n (\%) } \\
\hline Medicare & 391 (30.9\%) \\
\hline Medicaid & $176(13.9 \%)$ \\
\hline Private insurance & $611(48.4 \%)$ \\
\hline Self-pay & $34(2.7 \%)$ \\
\hline Other & $53(4.2 \%)$ \\
\hline \multicolumn{2}{|l|}{ Bed size of hospital, $n(\%)$} \\
\hline Small & $28(2.2 \%)$ \\
\hline Medium & $149(11.8 \%)$ \\
\hline Large & 1087 (86.0\%) \\
\hline \multicolumn{2}{|l|}{ Location and teaching status, $\mathrm{n}(\%)$} \\
\hline Rural & $30(2.4 \%)$ \\
\hline Urban non-teaching & $92(7.3 \%)$ \\
\hline Urban Teaching & 1142 (90.3\%) \\
\hline \multicolumn{2}{|l|}{ Region of hospital, n (\%) } \\
\hline Northeast & $342(27.0 \%)$ \\
\hline
\end{tabular}

\begin{tabular}{|c|c|}
\hline Midwest & $206(16.3 \%)$ \\
\hline South & 369 (29.2\%) \\
\hline West & $348(27.5 \%)$ \\
\hline \multicolumn{2}{|l|}{ Disposition of patient, $\mathrm{n}(\%)$} \\
\hline Routine & 207 (16.4\%) \\
\hline Transfer to short-term hospital & $110(8.7 \%)$ \\
\hline Transfer to nursing home & $109(8.6 \%)$ \\
\hline Home healthcare & $165(13.0 \%)$ \\
\hline Elective admissions, $\mathrm{n}(\%)$ & $233(18.5 \%)$ \\
\hline Died during hospitalization, $\mathrm{n}(\%)$ & $674(53.3 \%)$ \\
\hline Length of stay, days $(95 \% \mathrm{Cl})$ & $33.0(10.0,65.0)$ \\
\hline Cost, \$ & $319588.4 \pm 248995.8$ \\
\hline \multicolumn{2}{|l|}{ Complications, n (\%) } \\
\hline Transfusion & $292(23.1 \%)$ \\
\hline Gastrointestinal bleed & $149(11.8 \%)$ \\
\hline Hemoperitoneum & $35(2.8 \%)$ \\
\hline Intracranial bleed & $109(8.6 \%)$ \\
\hline Hemolytic anemia & $29(2.3 \%)$ \\
\hline Vascular complication & $41(3.3 \%)$ \\
\hline Acute renal failure & $998(78.9 \%)$ \\
\hline Dialysis & $234(18.5 \%)$ \\
\hline Mechanical complication & $47(3.8 \%)$ \\
\hline Systemic embolism & $197(15.6 \%)$ \\
\hline $\begin{array}{l}\text { Post-op stroke or transient ischemic } \\
\text { attack }\end{array}$ & $49(3.9 \%)$ \\
\hline Implant-related infection & $58(4.6 \%)$ \\
\hline Post-op wound infection & $45(3.6 \%)$ \\
\hline
\end{tabular}

\section{Time trends in utilization, mortality, cost, and LOS among BiVAD patients}

Between 2009 and 2015, the utilization of all BiVADsdecreased significantly $(-4.8 \%$ per year, $p<$ 0.001 , Figure 1). There was a significant increase in the use of internal BiVADs $(+10.0 \%$ per year, $p<$ 0.001 , Figure 1) and a significant decrease in the use of external BiVADs $(-9.4 \%$ per year, $p<0.001$, Figure 1$)$. There were no significant changes in mortality for all BiVADs, internal BiVADs, or external BiVADs (Appendix A). The costs associated with BiVAD implantation increased significantly $(+23.0 \%$ per year, $p<0.001)$. The LOS also increased, although not significantly $(p=$ 0.083 , Figure 2). Among complications associated with BiVAD implantations, the rates of hemoperitoneum $(p=$ $0.003)$, gastrointestinal ( $G I)$ bleed $(p=0.001)$, and acute renal failure $(p=0.008)$ increased significantly over time (Supplemental Table 1). Among known comorbidities, CAD ( $p=0.006), M I(p=0.010)$, fluid and electrolyte disorders $(p=0.043)$, and history of renal failure $(p=$ 0.011 ) increased in prevalence over time (Supplemental Table 1). 


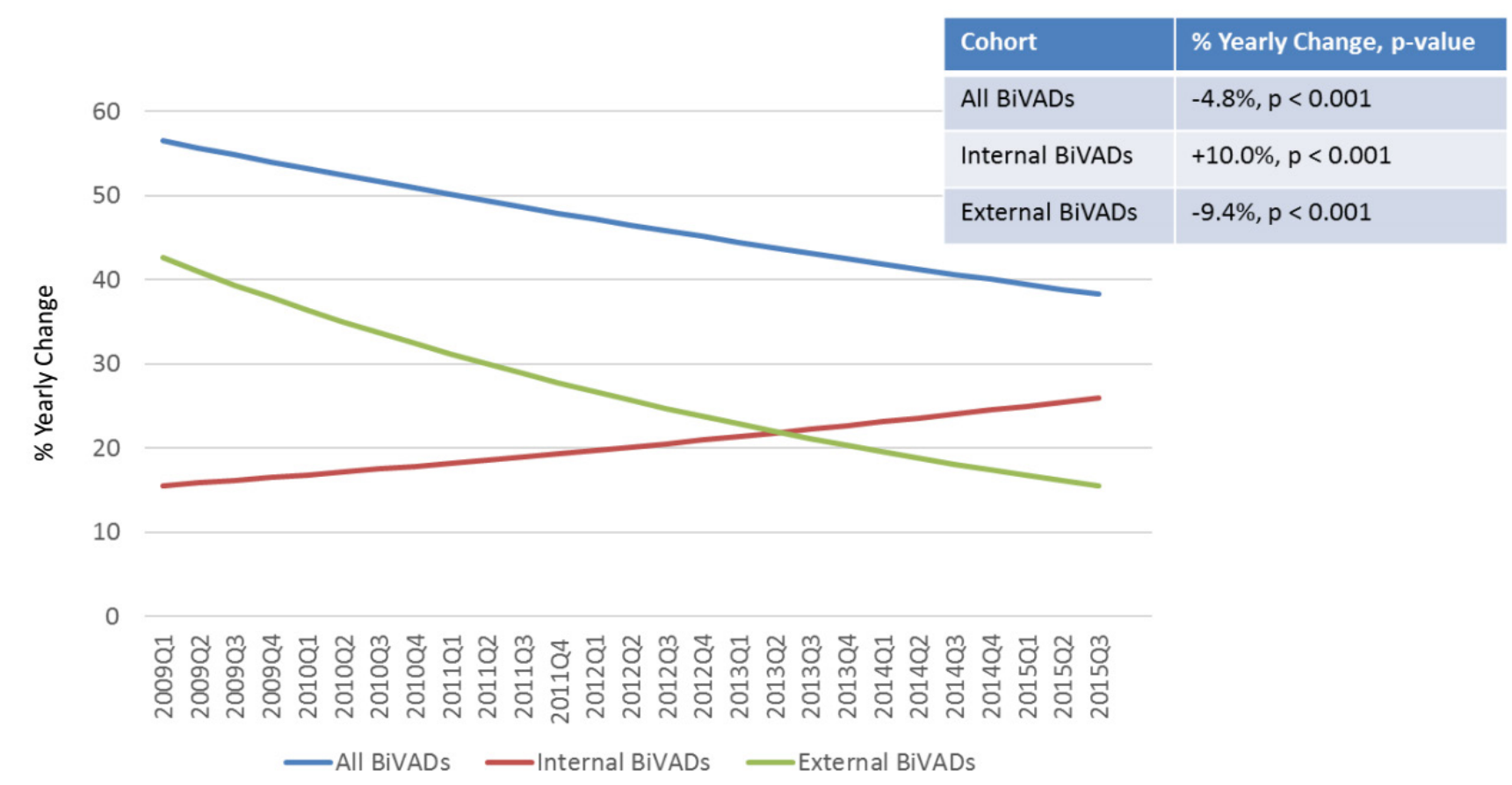

Figure 1: Time trend in utilization of internal, external, and all biventricular assist devices.

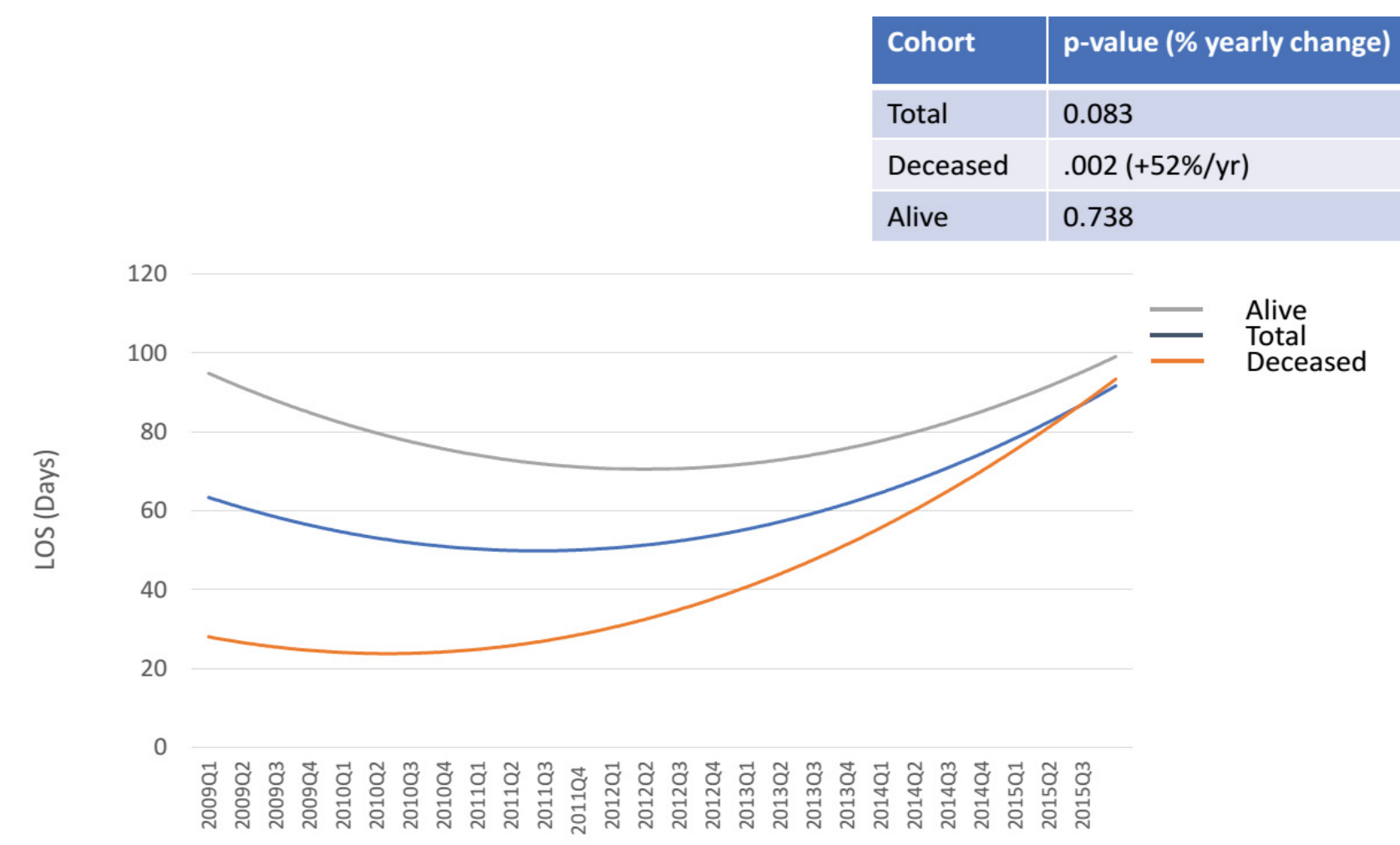

Figure 2: Trend in length of stay among all patients with biventricular assist devices, those who died during hospitalization, and those who survived.

Time trend analysis for cost and LOS among BiVAD patients: The survivors versus deceased

To explain the increase in cost and LOS among BiVAD patients despite no changes in mortality, an exploratory time trend analysis was performed. Stratification based on internal vs. external BiVADs demonstrated no significant time trends in cost and LOS. Stratification based on survival of the index hospitalization demonstrated no significant time trend in LOS or cost among those that survived, however, there was a significant and sizeable increase in LOS $(+52.0 \%$ per year, $p=0.002$, Figure 2$)$, as well as in cost $(+15 \%$ per year, $p<0.001$, Figure 3) among those that died. Even after controlling for known complications and comorbidities outlined in Supplemental Table 1, LOS (+3.8\% per year, 


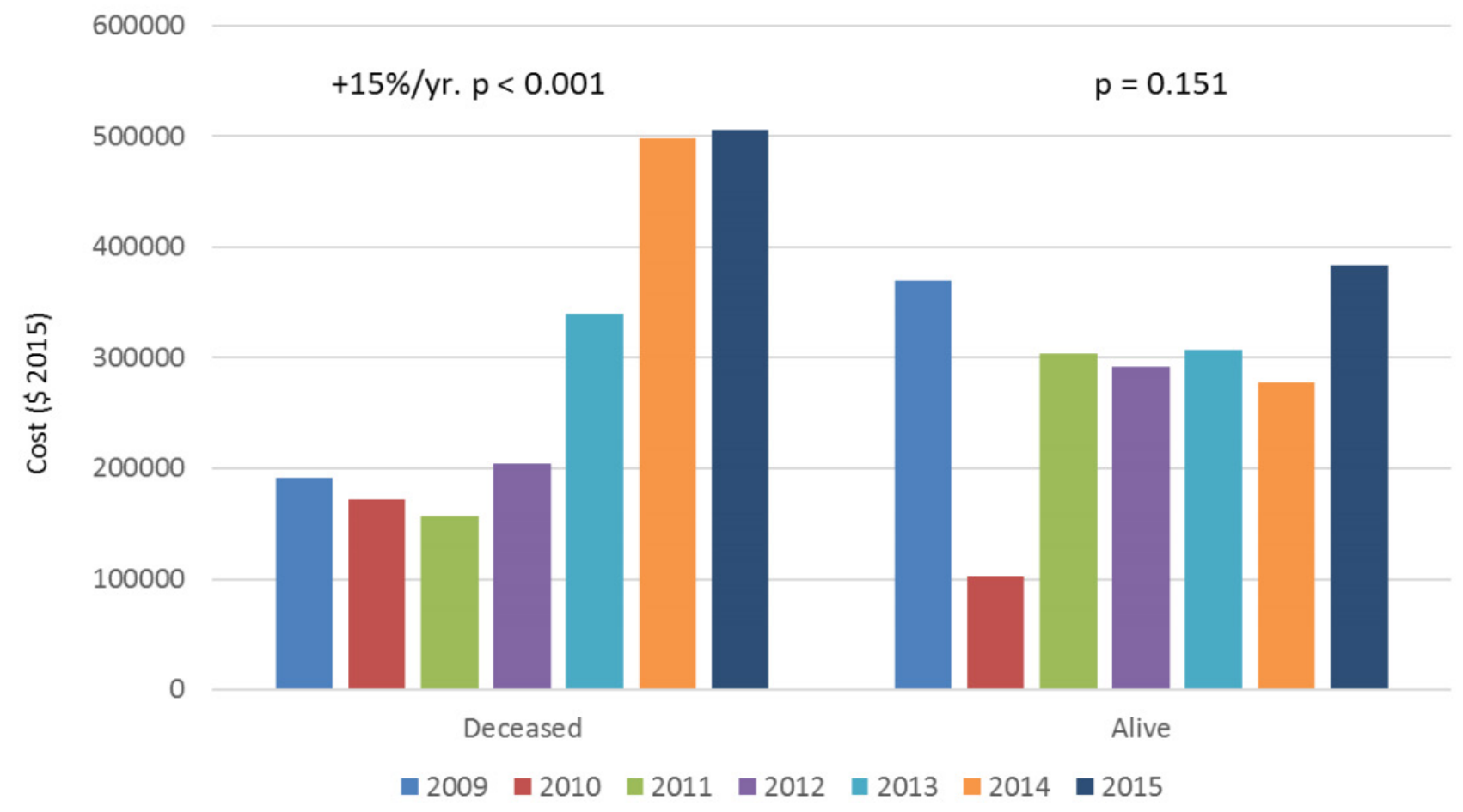

Figure 3: Trend in cost of hospitalization for those with biventricular assist devices who died during hospitalization, and those who survived.

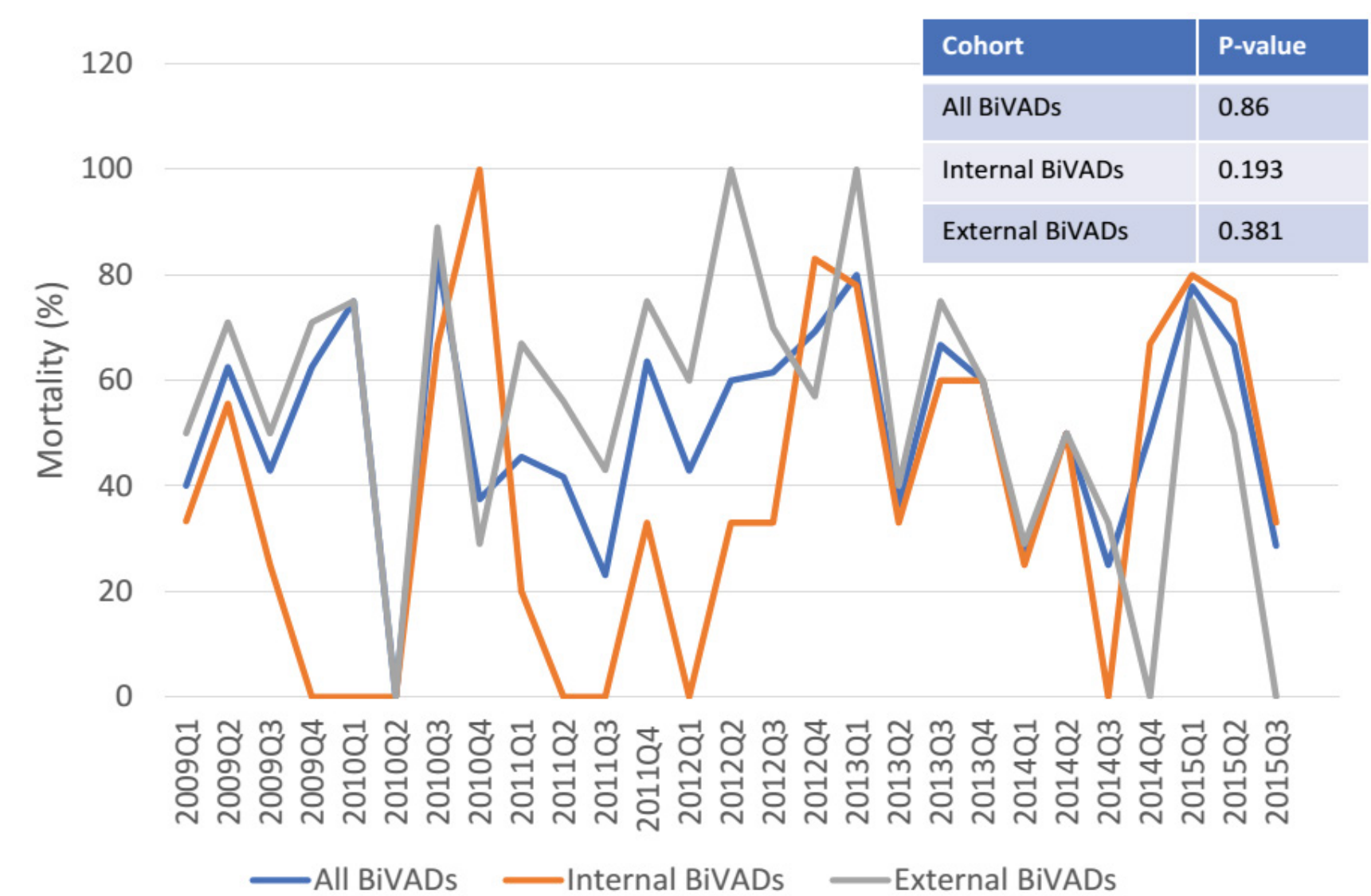

Appendix A: Trend in mortality of internal, external, and all biventricular assist devices.

$p=0.025)$ and cost $(+\$ 33,736$ per year, $p=0.002)$ increased significantly among the patients who died during their hospitalization. These results suggest that the increase in cost and LOS associated with all BiVAD scould be attributed to the substantial increase in these parameters among patients who do not survive the index hospitalization. 


\section{Comparisons between internal and external BiVADs: utilization, mortality, LOS, cost, and complications}

Internal BiVADs were implanted in 538 (42.5\%) patients and external BiVADs in 727 (57.5\%) patients (Table 2). More patients were female in the external
BiVAD group ( $30.1 \%$ vs. $18.4 \%, p=0.024)$. There were no significant differences between internal and external BiVADs in insurance coverage, size, and teaching status of the hospitals, as well as elective cases. More of the external BiVAD implantation occurred in the Northeast compared to the internal BiVADs $(36.0 \%$ vs. $14.9 \%, p=$

Table 2: Comparisons between internal and external BiVADs: utilization, mortality, length of stay, cost, and complications.

\begin{tabular}{|c|c|c|c|}
\hline & Internal BiVAD ( $n=538)$ & External BiVAD $(n=727)$ & $p$ value \\
\hline Age, years (standard deviation) & $49.5(13.2)$ & $52.4(15.2)$ & 0.098 \\
\hline Female, n (\%) & 99 (18.4\%) & 219 (30.1\%) & 0.024 \\
\hline \multicolumn{3}{|l|}{ Race, n (\%) } & \multirow[t]{8}{*}{0.03} \\
\hline White & 312 (66.9\%) & 407 (61.5\%) & \\
\hline Black & 89 (19.0\%) & 101 (15.2\%) & \\
\hline Hispanic & $40(8.6 \%)$ & $55(8.3 \%)$ & \\
\hline Asian or Pacific Islander & $11(2.3 \%)$ & $29(4.4 \%)$ & \\
\hline Native American & $5(1.0 \%$ & $5(0.8 \%)$ & \\
\hline Other & $10(2.1 \%)$ & 65 (9.9\%) & \\
\hline Missing & $72(13.4 \%)$ & $64(8.8 \%)$ & \\
\hline \multicolumn{4}{|l|}{ Comorbidities, n (\%) } \\
\hline Coronary artery disease & 119 (22.1\%) & 327 (44.9\%) & $<0.001$ \\
\hline Myocardial infarction & $93(17.4 \%)$ & 299 (41.2\%) & $<0.001$ \\
\hline Chronic pulmonary disease & $34(6.3 \%)$ & $78(10.7 \%)$ & 0.236 \\
\hline Iron deficiency anemia & $70(12.9 \%)$ & $102(14.1 \%)$ & 0.788 \\
\hline Chronic blood loss anemia & $10(1.9 \%)$ & $5(0.7 \%)$ & 0.386 \\
\hline Coagulopathy & 250 (46.5\%) & $356(49.0 \%)$ & 0.765 \\
\hline Diabetes & 79 (14.7\%) & 144 (19.8\%) & 0.269 \\
\hline Hypertension & $148(27.5 \%)$ & $228(31.4 \%)$ & 0.438 \\
\hline Hypothyroidism & $15(2.8 \%)$ & $39(5.4 \%)$ & 0.31 \\
\hline Liver disease & $30(5.5 \%)$ & $20(2.8 \%)$ & 0.242 \\
\hline Lymphoma & $10(1.9 \%)$ & $24(3.3 \%)$ & 0.463 \\
\hline Fluid and electrolyte disorders & $356(66.2 \%)$ & 435 (59.9\%) & 0.271 \\
\hline Metastatic cancer & $0(0.0 \%)$ & $5(0.6 \%)$ & 0.393 \\
\hline Obesity & 65 (12.0\%) & $63(8.7 \%)$ & 0.5 \\
\hline Peripheral vascular disorders & $45(8.4 \%)$ & 77 (10.6\%) & 0.517 \\
\hline Pulmonary circulatory disorders & $0(0.0 \%)$ & $38(5.3 \%)$ & 0.014 \\
\hline Renal failure & $194(36.1 \%)$ & 117 (16.1\%) & $<0.001$ \\
\hline Valvular disease & $0(0.0 \%)$ & $52(7.2 \%)$ & 0.003 \\
\hline \multicolumn{3}{|l|}{ Insurance, n (\%) } & \multirow[t]{6}{*}{0.768} \\
\hline Medicare & $172(31.9 \%)$ & $219(30.1 \%)$ & \\
\hline Medicaid & $44(8.3 \%)$ & $131(18.0 \%)$ & \\
\hline Private insurance & $298(55.5 \%)$ & $313(43.1 \%)$ & \\
\hline Self pay & $9(1.7 \%)$ & $25(3.4 \%)$ & \\
\hline Other & $14(2.6 \%)$ & $39(5.4 \%)$ & \\
\hline \multicolumn{3}{|l|}{ Bed size of hospital, n (\%) } & \multirow[t]{4}{*}{0.119} \\
\hline Small & $9(1.8 \%)$ & $18(2.5 \%)$ & \\
\hline Medium & $43(7.9 \%)$ & $107(14.7 \%)$ & \\
\hline Large & 486 (90.3\%) & $601(82.8 \%)$ & \\
\hline
\end{tabular}




\begin{tabular}{|c|c|c|c|}
\hline \multicolumn{3}{|l|}{ Location and teaching status, $\mathrm{n}(\%)$} & \multirow[t]{4}{*}{$<0.001$} \\
\hline Rural & $0(0.0 \%)$ & $30(4.1 \%)$ & \\
\hline Urban non-teaching & $9(1.7 \%)$ & $83(11.4 \%)$ & \\
\hline Urban Teaching & $528(98.3 \%)$ & $614(84.4 \%)$ & \\
\hline \multicolumn{3}{|l|}{ Region of hospital, n (\%) } & \multirow[t]{5}{*}{0.002} \\
\hline Northeast & 80 (14.9\%) & $262(36.0 \%)$ & \\
\hline Midwest & $111(20.7 \%)$ & $94(13.0 \%)$ & \\
\hline South & $169(31.4 \%)$ & $200(27.6 \%)$ & \\
\hline West & $178(33.0 \%)$ & $170(23.4 \%)$ & \\
\hline \multicolumn{3}{|l|}{ Disposition of patient, $\mathrm{n}(\%)$} & \multirow[t]{5}{*}{0.026} \\
\hline Routine & 127 (23.7\%) & $80(11.0 \%)$ & \\
\hline Transfer to short-term hospital & $24(4.4 \%)$ & $86(11.8 \%)$ & \\
\hline Transfer to nursing home & $45(8.4 \%)$ & $64(8.8 \%)$ & \\
\hline Home healthcare & $98(18.2 \%)$ & $67(9.2 \%)$ & \\
\hline Elective admissions, $\mathrm{n}(\%)$ & 77 (14.3\%) & $157(21.6 \%)$ & 0.119 \\
\hline Died during hospitalization, $\mathrm{n}(\%)$ & $244(45.4 \%)$ & 430 (59.1\%) & 0.022 \\
\hline Length of stay, days (95\% Cl) & $64.0(32.0,119.0)$ & $20.0(6.0,36.0)$ & $<0.001$ \\
\hline Cost, \$ & $468321.3 \pm 286928.0$ & $208809.9 \pm 134796.6$ & $<0.001$ \\
\hline \multicolumn{4}{|l|}{ Complications, n (\%) } \\
\hline Transfusion & 107 (19.9\%) & $185(25.4 \%)$ & 0.396 \\
\hline Gastrointestinal bleed & 75 (14.0\%) & 74 (10.1\%) & 0.351 \\
\hline Hemoperitoneum & $20(3.7 \%)$ & $15(2.1 \%)$ & 0.414 \\
\hline Intracranial bleed & $50(9.3 \%)$ & $59(8.2 \%)$ & 0.738 \\
\hline Hemolytic anemia & 24 (4.5\%) & $5(0.7 \%)$ & 0.038 \\
\hline Vascular complication & $15(2.8 \%)$ & $26(3.6 \%)$ & 0.789 \\
\hline Acute renal failure & $460(85.6 \%)$ & $538(74.0 \%)$ & 0.02 \\
\hline Dialysis & $126(23.4 \%)$ & $108(14.9 \%)$ & 0.088 \\
\hline Mechanical complication & $15(2.7 \%)$ & $33(4.5 \%)$ & 0.43 \\
\hline Systemic embolism & $110(20.4 \%)$ & $88(12.1 \%)$ & 0.072 \\
\hline Post-op stroke or transient ischemic attack & $25(4.6 \%)$ & $24(3.3 \%)$ & 0.605 \\
\hline Implant-related infection & $35(6.4 \%)$ & $23(3.2 \%)$ & 0.432 \\
\hline Post-op wound infection & $25(4.7 \%)$ & $20(2.7 \%)$ & 0.405 \\
\hline
\end{tabular}

0.002). Internal BiVAD implantations were more likely than external BiVAD implantations to take place in urban teaching hospitals ( $98.3 \%$ vs. $84.4 \%, p<0.001$ ). Significantly more patients in the external BiVAD group had documented history of CAD ( $44.9 \%$ vs. $22.1 \%$, p < $0.001)$, pulmonary circulatory disorders $(5.3 \%$ vs. $0, p$ $=0.014)$, and valvular disease $(7.2 \%$ vs. $0, p=0003)$. History of renal failure was more prevalent in the internal BiVAD group ( $36.1 \%$ vs. $16.1 \%, \mathrm{p}<0.001$ ).

Higher mortality was observed among patients with external BiVADs compared to those with internal BiVADs (59.1\% vs. $45.4 \%, p=0.022$, Table 2$)$. Internal BiVADs were associated with longer median LOS (64.0 vs. 20.0 days, $p<0.001)$ and higher cost $(\$ 468,321$ vs. $\$ 208,809$ per hospitalization, $p<0.001)$. There were more complications of acute renal failure $(85.6 \%$ vs. $74.0 \%, p=0.020)$ and hemolytic anemia ( $4.5 \%$ vs. $0.7 \%$, $p=0.038$ ) among those with internal BiVADs. There were no significant differences in rates of bleeding, infection, or stroke.

\section{Discussion}

This study is the first to describe national trends in utilization, mortality, LOS, and cost for patients receiving surgically implanted internal and external BiVADs. From 2009 to 2015, the utilization of all BiVADs nationwide decreased despite an increase in the utilization of internal BiVADs as a result of the dramatic decrease in external BiVAD utilization. Compared to the significant decline in mortality and cost associated with LVAD implantation during this period [1,2], our analysis demonstrated no change in mortality and a rise in cost and LOS associated with BiVADs. The burden of comorbidities associated with the patients who have undergone BiVAD implantation increased significantly during this period. While there was no significant time trend in LOS and the cost of hospitalization for patients 
who survived the index hospitalization, LOS and costs related to the deceased patients increased, even after controlling for all comorbidities and complications. These findings suggest that the increase in LOS and cost are mostly driven by the patients who did not survive the index hospitalizations. One potential explanation for these trends is the increase in the burden of comorbidities of patients who receive BiVADs. These trends may be a testament to our evolving capability to care for higher-risk patients, which in turn, lead to higher costs. This study raises awareness of the need for a national registry for BiVADs to share our experiences, improve upon the selection of patients who will benefit the most from this intervention, and optimize patient outcomes and healthcare cost-efficiency.

The decline in surgically implanted BiVAD utilization was driven by a decrease in the utilization of external BiVADs, which outnumbered the increase in the utilization of internal BiVADs. Our findings correlated with a prior study that used the UNOS database, which showed a decrease in utilization of PF-BiVADs from 2010 to 2013 [6]. Even though the reasons for the decrease in the utilization of surgically implanted external BiVADs are multifactorial and cannot be concluded from the NIS dataset, we can speculate a few potential reasons. First, the increase in utilization of percutaneous biventricular mechanical circulatory support, such as peripheral extracorporeal membrane oxygenation, and right ventricular support devices including Impella RP, and ProtekDuo, may be obviating the need for surgically placed BiVADs. Second, improvement in LVAD technology, optimization of patient selection, timely LVAD implantation, as well as improvement in perioperative management of the patients could have played a role in mitigating the RV failure and need for BiVAD implantation.

This is the first study to directly compare outcomes of surgically implanted internal and external BiVADs. Patients with internal BiVADs had significantly longer LOS and higher cost of hospitalizations compared to that of external BiVADs; however, the mortality was lower in patients with internal BiVADs. More studies are needed to understand the optimal and individualized BiVAD configuration for each patient, appropriate patient selection criteria for each configuration, and management strategies for better outcomes and costefficiency.

Despite the advancement of LVAD technology and patient care, RVF after LVAD placement remains a significant source of complication - upto $3.9-14.5 \%$ according to the recent multi-national studies $[7,8]$. Prior studies have demonstrated that planned, and earlier BiVAD implantation is associated with higher survival rates $[9,10]$. Other studies have demonstrated that angiotensin-converting enzyme inhibitorscan significantly reduce the risk of $\mathrm{GI}$ bleeds associated with
LVAD implantation [11] and improve mortality [12]. However, it is unclear whether these findings have led to changes in the management of patients with BiVAD. Future investigation should focus on implantation of evidence-based strategies for practical improvements in the care of patients with BiVADs.

\section{Limitations}

This study used ICD-9 codes to identify the population that received BiVADs, and thus, have limitations inherent to the coding system. For example, the diversity in BiVAD configurations, e.g., extracorporeal vs. implantable and pulsatile vs. continuous, temporary percutaneous support systems, and TAH, were not captured in this coding system. Thus, our study was not able to assess the differences in utilization and outcomes across the different configurations. Moreover, given the notable mechanical and hemodynamic differences among BiVAD configurations, the observed trends in our paper should be interpreted cautiously and cannot be used to infer conclusions regarding any individual BiVAD configuration. This study also does not include many other types of MCS, such as paracorporeal devices and other external systems. The differences in outcomes associated with the diverse BiVAD configurations is an important area for future research.

It should be also noted that the off-label use of twoseparate surgically implanted VADs used for biventricular support was classified as two single ventricular devices (ICD-937.65), andthus, wereexcluded from our study. Consequently, our study captures only a subset of internal BiVADs, and likely underestimates the total number of internal and all BiVADs. Despite these limitations, our study demonstrated a significant rise in internal BiVAD utilization during the study period. Future studies are needed to better understand the reason for the increase in utilization as well as the optimal patient selection criteria and peri-operative management strategies to improve outcomes.

Our study was limited to ICD-9 codes, which capture only three-quarters of the hospitalizations in 2015 (switched to ICD-10 thereafter). We addressed this limitation by evaluating time trends based on quarterly numbers. One of the limitations of the NIS database is that it does not allow for stratification of the data based on a center's volume of BiVAD implantations. Moreover, given that most centers that perform BiVADs were large-volume centers, this analysis was not powered to detect differences based on implantation experience. As an exploratory analysis, our study tested several hypotheses, which likely reduced the power of the study. This study is retrospective andour findings should be confirmed with prospective trials when possible.

\section{Conclusion}

From 2009 to 2015, BiVAD utilization decreased, driven by the dramatic decrease in surgically implanted 
external BiVAD utilization. Even though there was no change in mortality, the cost and LOS have increased in this period, mostly driven by the patients who did not survive the index hospitalization of implantation. In the same period, the burden of comorbidities has increased, suggestive of our evolving capability to care for sicker patients. Compared to patients with external BiVADs, patients with internal BiVADs had longer LOS and higher costs associated with their hospitalizations but were also more likely to survive. There is a need for a national registry to share experiences, and optimize the utilization of BiVAD to improve patient outcome and cost-efficiency.

\section{Conflict of Interest and Source of Funding Statement}

There are no funding sources or relevant financial relationships for all authors to disclose.

\section{Authors Contribution}

Each author has made substantial intellectual contribution to this work including contribution to conception, design, data acquisition, or analysis and interpretation. Each has also revised the manuscript, and approved this manuscript in its current form.

\section{References}

1. Briasoulis A, Inampudi C, Akintoye E, Adegbala O, Asleh $\mathrm{R}$, et al. (2018) Regional variation in mortality, major complications, and cost after left ventricular assist device implantation in the United States (2009 to 2014). Am J Cardiol 121: 1575-1580.

2. Shah N, Agarwal V, Patel N, Deshmukh A, Chothani A, et al. (2016) National trends in utilization, mortality, complications, and cost of care after left ventricular assist device implantation from 2005 to 2011. Ann Thorac Surg 101: 1477-1484.

3. Mehra MR, Uriel N, Naka Y, Cleveland Jr JC, Yuzefpolskaya M, et al. (2019) A fully magnetically levitated left ventricular assist device - Final Report. N Engl J Med 380: 1618-1627.
4. Dang NC, Topkara VK, Mercando M, Kay J, Kruger KH, et al. (2006) Right heart failure after left ventricular assist device implantation in patients with chronic congestive heart failure. J Heart Lung Transplant 25: 1-6.

5. Briasoulis A, Akintoye E, Mohsen A, Inampudi C, Briasouli $A$, et al. (2020) Trends in utilization, mortality, major complications, and cost after total artificial heart implantation in the United States (2009-2015). Hell J Cardiol 61: 407412.

6. Levin AP, Jaramillo N, Garan AR, Takeda K, Takayama $H$, et al. (2016) Outcomes of contemporary mechanical circulatory support device configurations in patients with severe biventricular failure. J Thorac Cardiovasc Surg 151: 530-535.e2.

7. Kiernan MS, Wilson Grandin E, Brinkley Jr M, Kapur NK, Thinh Pham D, et al. (2017) Early right ventricular assist device use in patients undergoing continuous-flow left ventricular assist device implantation: Incidence and risk factors from the interagency registry for mechanically assisted circulatory support. Circ Heart Fail 10: e003863.

8. Soliman OII, Akin S, Muslem R, Boersma E, Manintveld OC, et al. (2018) Derivation and validation of a novel right-sided heart failure model after implantation of continuous flow left ventricular assist devices: The EUROMACS (European Registry for Patients with Mechanical Circulatory Support) Right-Sided Heart Failure Risk Score. Circulation 137: 891906.

9. Fitzpatrick JR, Frederick JR, Hiesinger W, Hsu VM, McCormick RC, et al. (2009) Early planned institution of biventricular mechanical circulatory support results in improved outcomes compared with delayed conversion of a left ventricular assist device to a biventricular assist device. J Thorac Cardiovasc Surg 137: 971-977.

10. Shah P, Ha R, Singh R, Cotts W, Adler E, et al. (2018) Multicenter experience with durable biventricular assist devices. J Hear Lung Transplant 37: 1093-1101.

11. Converse MP, Sobhanian M, Taber DJ, Houston BA, Meadows HB, et al. (2019) Effect of Angiotensin II inhibitors on gastrointestinal bleeding in patients with left ventricular assist devices. J Am Coll Cardiol 73: 1769-1778.

12. Ghashghaei R, Yousefzai R, Liwinski F, Tran H, Adler ED (2016) The role of neurohormonal therapy in patients with left ventricular assist devices. J Hear Lung Transplant 35: S396. 